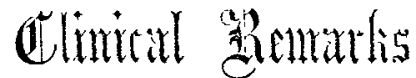

on

\section{THE USE OF STIMULANTS IN FEVER.*}

By W. WILLIAMSON, M.D.,

RXAMINER IN MEDICINE IN THE UNIVERSITX OF ABERDEEN, PHYSICIAY TO THE ABERDETS INFIRYAARY.

AT the present day there is, perhaps, no question of greater interest to the medical profession generally, and to hospital physicians in particular, than the use and abuse of stimulants in fever.

If Dr. Gairdner's opinion is a sound one-that " to give wine, whisky, and beef-tea, while withholding milk, is simply to destroy your patient; and the more wine or whisky you give, while withholding milk, the more sure you will be to destroy your patient soon, because you are thereby super- seding the natural appetite (or what remains of it) for a nourishing and wholesome diet, by a diet-if it can be so called-which poisons the blood and checks the excretions, and alters for the worse the whole tone of the nervous system, and of the digestion and assimilation,"-then we must give up the old practice of administering wine as the rule in fever, and adopt the new, of giving milk.

Dr. Kennedy has a strong impression that the practice of medicine has literally retrograded within the last few years. He seems alarmed at the amount of scepticism which prevails at present ; and he cannot refrain from expressing a feeling of disappointment at bleeding, blisters, purgatives, calomel, and tartar emetic and wine being abused and ignored, and the experience of men, who were indisputably not behind any now living, being utterly slighted.

This is a gloomy view to take of the present position of the medical art, and one assuredly not in accordance with the experience of the profession generally.

Dr. Haldane, in his recent excellent lecture at the College of Surgeons, Edinburgh, gave it as his opinion that an extraordinary advance had been made in pathology and diagnosis; and that a more rational method of treatment was pursued, inasmuch as " the physician seldom attempts to cure disease, but endeavours to place and maintain his patient in the most favourable condition for recovery."

That the treatment of disease has neither stood still nor retrograded, but progressed, is the belief of the majority of the medical profession. This is in a great measure owing to an advanced pathology, but also to the existence of a certain amount of healthy scepticism. In Dublin they do not use wine because they have fever to treat, but because the case requires it. Why, then, should they bleed and blister, give calomel and tartar emetic in diseases merely because experienced men used to do so? We ought to think for ourselves, and not blindly pin our faith to any authority, however great.

If Dr. Gairdner can satisfactorily show that fever can be treated by a method apparently the opposite to that at present followed, with a diminished rate of mortality, it behoves us cautiously, but honestly, to investigate the truth of his statements, and to try whether they will stand the test of experience. Should they be proved to be true, not only would our patients thereby be benefited, but (not an unimportant consideration) a very considerable saving would be effected in one item of hospital expenditure. In the Aberdeen Infirmary the account for wine and spirits in 1862, when there was no epidemic of fever, amounted to $£ 100$, while last year, when the

* This valuable paper has a peculiar and melancholy interest from the circumstances attending the recent and premature death of its lamented author from that disease of which this lecture treats. He was at the time engaged in the study and treatment of cases of typhus in the course of his duty; and the manuscript bears date the 3rd of February, the very day he was seized with the premonitory symptoms of typhus from which he died at the end of a fortnight. They are the latest words of a faithfin labourer in his vocation, who met his death in combating that fatal disease of which he writes so well. - En, $\mathrm{L}$.

No. 2166. town was visited by a serere epidemic, it reached the sum of £325. Now if Dr. Gairdner is correct in saring "that intinite mischief has been done in typhus fever, and in all fevers, by giving wine, and withholding or not giving milk, it will be readily perceived what an enormous saving would be effected annually in the hospitals of the United Kingdom. On one point we may feel assured, that such an inquiry as Dr. Gairdner is conducting will be followed ont by him in an impartial and scientific spirit.

In THE LANCET of the 28th of January we have a report of twelve cases of typhus and typhoid fever treated without stimulants at Guy's Hospital by Dr. Wilks, and from a perusal of this paper I have been led to consider how far the views advanced by Dr. Gairdner are borne ont by the treatment adopted in these cases. The title of the paper would naturally lead one to suppose that no stimulant had been given in these twelve cases, whereas the reports of the individual cases show that stimulants were administered in several. The number of the cases may be reduced to eleven, as there is no evidence to show that Case 12 was typhoid fever, and, besides, it is imma. terial to the question of treatment, as the patient did not recover. Of these eleven cases wine was given in three, and brandy in a fourth; so that stimulants were given in more than a tinird of the cases.

Case 6 is headed " Typlus fever treated without stimulants," yet we find that six ounces of wine were given, though it is added, "for drink." Would not cold water have done equally well ?--for no beverage is more grateful to a fever patient.

Case 7, though entitled "Typhus fever," reads more like a case of typhoid, from the fact of fresh rose spots appearing. Here also wine was given.

Case 8 seems to have been a severe one, venesection having been employed to the extent of twelve ounces in consequence of an attack of hemoptysis. This case was at first treated without wine, but a relapse having taken place, ammonia and wine were ordered.

Case 11. "Typhoid fever ; slight perforation (?); recovery." It was believed that perforation of the bowel had taken place, though, as the author remarks, "the recovery would almost preclude such a supposition, especially in the alsence of evidence of general peritonitis." In this case the symptoms were of the gravest nature, the pulse nearly 200 , and the belief was that the boy would shortly die. He recovered, however, and he seems to have owed his life to turpentine fomentations and brandy.

This report cannot be considered a satisfactory contribution towards the settlement of the question as to the treatment of ferer without stimulants.

Another point in the report requires notice. Dr. Wilks says, "for whilst we are witnessing the free use of stimulants in fever, we are reading that it was Dr. Graves's plan to administer antimony in the same disease." The inference from this would appear to be that Dr. Graves gave no wine, but antimony instead. The reader would, however, form a very erroneous opinion regarding Dr. Graves's practice were he to rest in such a belief. The fact is, Dr. Graves rarely gave antimony alone -it was almost invariably combined with opium; and the combination proved a valuable medicine in the class of eases in which it was administered-namely, those characterized by great excitement of the nervous system. But Dr. Graves seldom trusted to antimony and opium: he frequently gave wine; and perhaps no writer is more particular in the rules he lays down with reference to wine in fever. He also gave porter, and, above all, he fed fevers. He acknowledges that the treatment by antimony and opium occasionally disappointed him, when he calculated upon its succeding.

With regard to the difference of upimion widely prevailing in the ranks of the medical profession as to the reason why diseases are now treated differently from formerly, sufficient proof has not yet been adduced in farour of a change of the type of disease, or of a deterioration of the human constitution. Is not the opinion expressed by Dr. Jemner nearest the truth?- that diseases preserve their essential characters and natures from age to age, while the opinions of the profession respecting them and their treatment change from yea: to year - the result sometimes of the personal sway of some influential teacher, sometimes of real advances in pathology and therapeutics.

The rules of treatment in fever laid down by Dr. Wilks are similar to those followed in the Aberdeen Infirmary. We believe support and a moderate amount of wine to be best; and nothing would seem sounder than the opinion expressed by the pinsicians of Gay"s Hospital, "that a large number of cases ran their course without any other treatment than care- 
ful watching and feeding, but that some needed a stimulant during the progress of the disease, and others required it from the commencement." If it had been added that the first class of cases occurred mostly in young persons, the opinion arrived at by these able physicians would have coincided with that entertained by my colleagues in this in irmary. Young persons, says Dr. Wilks, always do well if left alone. I cordially agree with this statement; for ont of a number of cases occurring in young persons under the age of fourteen years, in only one was wine given, and to the extent of not more than two ounces daily. In the worst cases, in those adranced in life, we never gave more than eight ounces of brandy and four ounces of wine in twenty-four hours; and this quantity was considered excessive, and not given in more than three per cent. of the cases treated.

\section{CLINICAL NOTES}

or

UTERINE S URGER Y.

Br J. MARION SIMS, M.D.,

SLRGEON TO THE WOMAN'S HOSPITAL, NEW YORK.

No. III.

PAINFUL MIENSTRUATION.

Menstruation may be attended by a general malaise, but should not, as a rule, be accompanied by any very severe degree of suffering. If there is much pain, either preceding its irruption or during the flow, there will always be a physical condition to account for it, and this will be of a nature to obstruct mechanically the egress of the fluid from the cavity of the womb. The obstruction may be the result of inflammation and attendant turgescence of the cervical mucous membrane, whereby this canal becomes narrowed merely by the tumefaction of its lining coat. But the most frequent cause of obstruction is purely anatomical and mechanical. For instance, the os and cervix uteri may be preternaturally small, or the canal may be flexed; or these may be complicated with the presence of a polypus, or with that of a fibroid tumour, in either the anterior or posterior wall of the uterus, and occasionally in the antero-lateral portion.

Oi 250 married women who had never borne children, 129, or more than hale, had pain of an abnormal kind attending the menstrual flow. I have been in the habit of dividing these into two classes, calling the one painful, and the other excessively painful, or dysmenorrhoeal. Of these 129, 100 were painful, or 1 in $2 \frac{1}{2}$ of the whole number; 29 were dysmenorrhoeal, or 1 in $8 \frac{6}{10}$. Of the 100 painful menstruations, 58 had anteversion, or more properly speaking, anteflexion; 17 of these had fibroid tumours in the anterior wall : 25 had retroversion; 7 of these had fibroid tumours in the posterior wall: and in 17 the position was normal, 1 of these haring a fibroid tumour in the fundus. Of the 29 dysmenorrhoal cases, 23 had anteversion; 14 of these had fibroid tumours in the anterior wall : 3 had retroversion; all of these had fibroid tumours in the posterior wall: and in 3 the position was normal. Of the 100 cases of painful menstruation, the os was normal in but 6 , unnaturally contracted in 90, otherwise abnormal in 4 . Of the 29 cases of dysmenorrhcea, properly speaking the os was not normal in a single case, being contracted in 26 , and otherwise abnormal in the other 3 .

The following tabular statement presents the particulars at a glance.

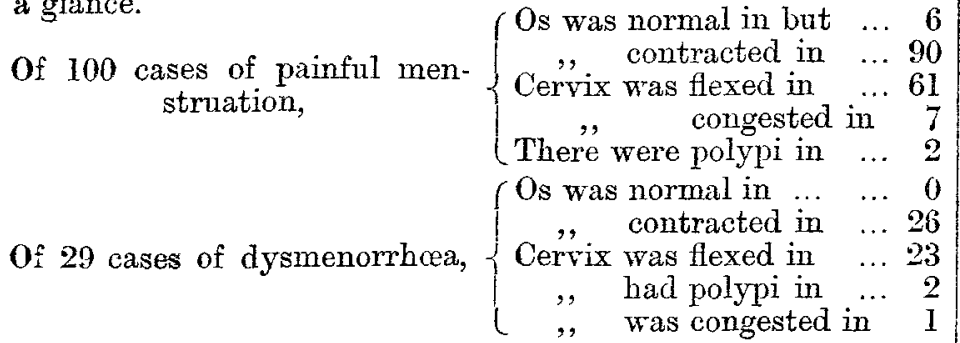

From this it would appear that the pain of menstruation is almost wholly due to mechanical causes, for of the whole 129 ouly 8 had engorgement or congestion of the lining membrane of the canal of the cervix, and these were mostly complicated either with flexure of the cervix or with fibroid growths in some portion of the body of the uterus. I would not deny that menstruation may be painful merely from a congested state of the cervical membrane, where there is no fibroid growth, no polypus, no contracted os, and no flexure of the cervix; but such cases are very rare indeed, while the great majority of dysmenorrhœal cases have a contracted os and a narrowed cerrical canal or a flexed one. In some instances the os is not larger than a pin's head, or it may be large enough to admit a No. 4 bougie. Again, the os may be quite large enough, but the canal may be flexed so as to form a valvular obstruction to the egress of the menstrual fluid. Sometimes we find the os small and the canal flexed without painful menstruation, and here the cervix is not indurated, but soft and elastic to the touch. Of the 129 cases of painful menstruation, but 20 had the uterus in its normal position, while 81 had anteversion (31 of these with fibroids in anterior wall), 28 retroversion (10 of these with fibroids), 116 had contracted os, and but 6 a normal one; while 84 had flexure of the cervical canal, 4 had polypi, and 8 engorgement of os and cervical mucous membrane.

In the great majority of cases, in addition to a contraction or flexure of the canal, the cervix will be long, pointed, and indurated. If the flexure be anteriorly, we often find the intra. vaginal portion of the cervix unequally developed-that is, the posterior part, from the os to the insertion of the vagina at $a$ (Fig. 14) may be an inch and a quarter long, while the anterior, from the os to the insertion of the anterion cul de sac at $b$, may not be more than one-third as long. But if there be a retroversion, then the os may be sucker-mouthed, the anterior lip overlapping the posterior, as in Fig. 15.

The size of the os and the position and relations of the cervix may be ascertained by the touch, as already explained. But it is

FIG. 14.

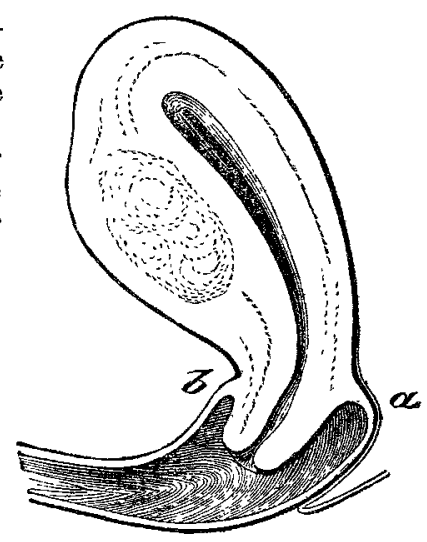

well always to resort to the sound to determine definitely the course, curvature, and contraction of the canal. To the touch and the sight the os may seem to be quite large enough, and

FIG. 15 .

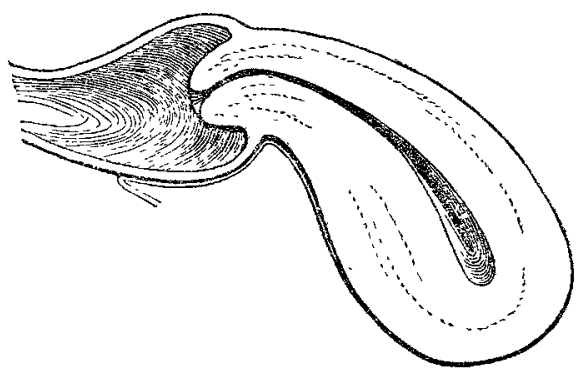

then we will find a flexure, perhaps a very acute one, at the junction of the cervix and body of the womb, due most probably to the pressure of a small fibroid in the anterior wall of the uterus, such as is represented by Fig. 16, a. But, leaving malpositions of the organ for the present, we will confine our-

Fig. 16.

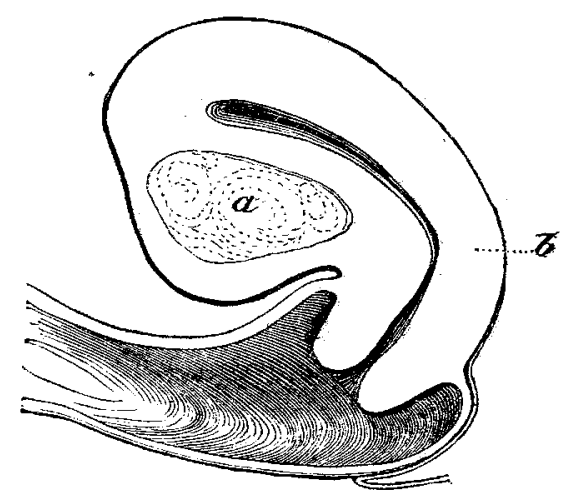

selves to ach cases as have a contracted camal or a flexed

* THE LA.TCET, Oct. 8th, 1864, p. 414 . 